\title{
A multi-wavelength analysis of the WASP-12 planetary system
}

\author{
Luca Fossati ${ }^{1}$, Carole A. Haswell ${ }^{1}$ and Cynthia S. Froning ${ }^{2}$ \\ ${ }^{1}$ Department of Physics and Astronomy, Open University, \\ Walton Hall, Milton Keynes MK7 6AA, UK \\ email: 1.fossati@open.ac.uk, C.A.Haswell@open.ac.uk \\ ${ }^{2}$ Center for Astrophysics and Space Astronomy, University of Colorado, \\ 593 UCB, Boulder, CO 80309-0593, USA \\ email: cynthia.froning@colorado.edu
}

\begin{abstract}
WASP-12 is a 2 Gyr old solar type star, hosting WASP-12b, one of the most irradiated transiting planets currently known. We observed WASP-12 in the UV with the Cosmic Origins Spectrograph (COS) on HST. The light curves we obtained in the three covered UV wavelength ranges, all of which contain many photospheric absorption lines, imply effective radii of $2.69 \pm 0.24 \mathrm{R}_{J}, 2.18 \pm 0.18 \mathrm{R}_{J}$, and $2.66 \pm 0.22 \mathrm{R}_{J}$, suggesting that the planet is surrounded by an absorbing cloud which overfills the Roche lobe. We clearly detected enhanced transit depths at the wavelengths of the MgII h\&k resonance lines. Spectropolarimetric analysis of the host star was also performed. We found no global magnetic field, but there were hints of atmospheric pollution, which might be connected to the very unusual activity of the host star.
\end{abstract}

Keywords. ultraviolet: stars, stars: magnetic fields, stars: individual (WASP-12), stars: activity, stars: abundances

\section{Introduction}

Transiting exoplanets provide a huge amount of information on both properties and evolution of planets outside our solar system. In particular close-in exoplanets are good 'laboratories' to test and constraint models of exoplanet atmospheres and dynamical evolution. These objects undergo a very strong star-planet interaction to the extent that the stellar activity is influenced by the presence of the planet (e.g. Shkolnik et al. 2005) and the star controls the planet's life-time (Li et al. 2010; Fossati et al. 2010a).

WASP-12 is a solar-type star (F9V), hosting one of the hottest, most bloated known exoplanets. It has one of the shortest known orbital periods, and is extremely close to the host star (Hebb et al. 2009). WASP-12b provides a key test of theories of planet evaporation and star-planet interactions.

\section{The HST observations}

UV observations of HD 209458 b, the prototype transiting hot Jupiter, were of the farUV Ly $\alpha$ emission line. The hydrogen abundance makes this an attractive line to observe, but the temporal and spatial variability of stellar Ly $\alpha$ emission is a highly undesirable complicating factor. For this reason, and to obtain better signal to noise, we observed WASP-12 in the near-UV where there are many other resonance lines including the very strong MgII h\&k lines. Observations were performed with COS (see Osterman et al. 2011) on HST for five consecutive HST orbits on 2009 September 24th (see Fossati et al. (2010a) for more details on the data reduction). 

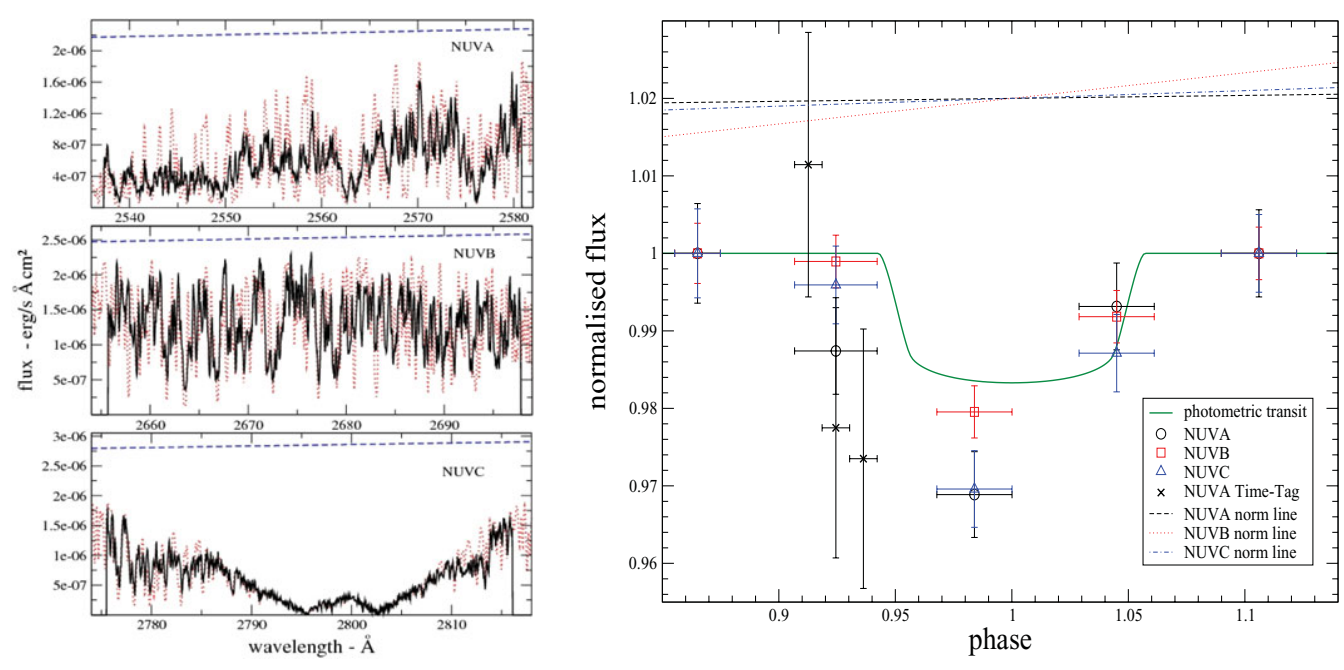

Figure 1. Left panel: Comparison between the observed fluxes of WASP-12 (full line) and LLMODELS synthetic fluxes (dotted line). The dashed line shows the modeled level of the stellar continuum flux. The three observed spectral ranges are defined as NUVA, NUVB and NUVC, from top to bottom. Right panel: Light curve obtained for each observed wavelength range (NUVA: open circles - NUVB: open squares - NUVC: open triangles). The horizontal error bar defines the orbital phase range covered by each observation. Vertical uncertainties come from a Poissonian treatment of the error bars. The full line shows the photometric transit light curve by Hebb et al. (2009). The three light curves were normalised to the line passing through the out-of-transit photometric points. The earlier ingress is shown by the crosses (from Fossati et al. 2010a).

The left panel of Fig. 1 shows the total summed spectrum in comparison with synthetic fluxes from the LLMODELS stellar model atmosphere code (Shulyak et al. 2006), assuming fundamental parameters and abundances by Fossati et al. (2010b).

The right panel of Fig. 1 compares the transit light curves obtained in each observed wavelength range with a fit to optical photometry (Hebb et al. 2009). As shown on the left panel of Fig. 1, the NUVB wavelength range is the closest to the stellar continuum and shows a transit depth that matches, at $\sim 1 \sigma$, the transit light curve derived from optical photometry. In the NUVA and NUVC wavelength ranges we obtained a deeper transit at $\sim 2.5 \sigma$ level. The NUVC spectral region is clearly dominated by the Mg II resonance lines, which are probably responsible for the detected extra depth in the transit light curve. On the other hand, the NUVA spectral region does not include dominant lines, but shows the presence of several resonance lines of various metals, in addition to $\mathrm{Mg} \mathrm{I}$ and Fe I lines from low energy levels, which dominate the stellar spectrum. These spectral features, formed mostly by neutral atoms, are likely to cause the observed deeper transit.

From the transit depth in each of the three observed wavelength ranges we obtained three values of the effective planet radius (NUVA: $2.69 \pm 0.24 \mathrm{R}_{J}, \mathrm{NUVB}: 2.18 \pm 0.18 \mathrm{R}_{J}$, and NUVC: $2.66 \pm 0.22 \mathrm{R}_{J}$ ), concluding that the radii obtained from the NUVA and NUVC wavelength regions exceed the planet Roche lobe $\left(2.36 \mathrm{R}_{J}\right)$, implying that WASP$12 \mathrm{~b}$ is currently losing mass.

\section{WASP-12 accretion disk and possible atmospheric pollution}

Fig. 1 shows that the NUVA flux during the second exposure lies below the out-oftransit level by $\sim 2 \sigma$. We divided this particular exposure into three equal sub-exposures, 

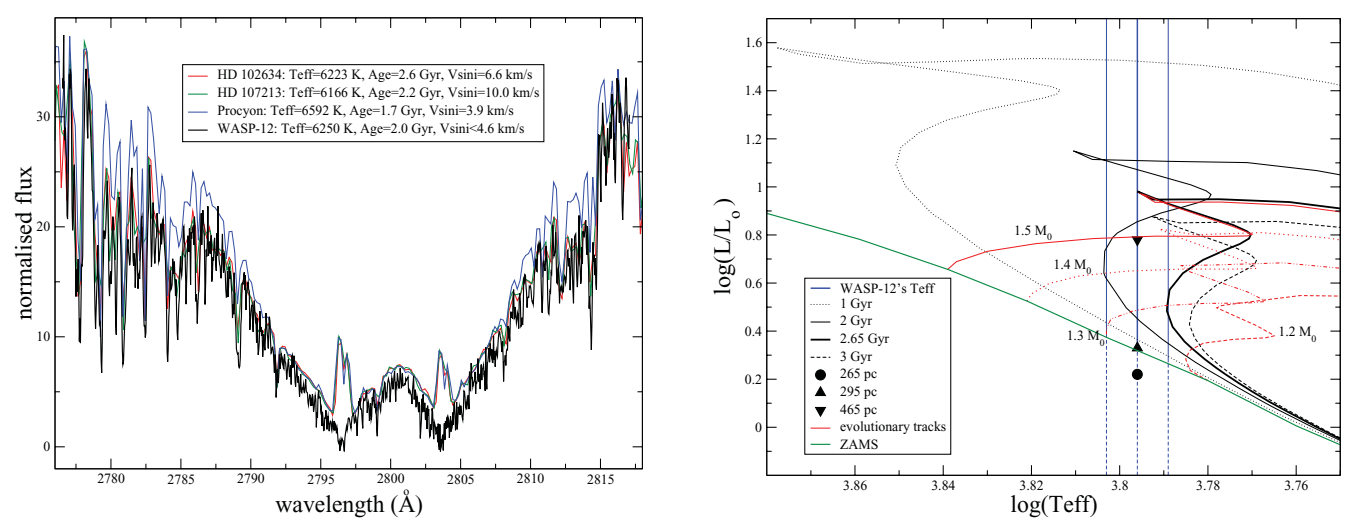

Figure 2. Left panel: Comparison of the Mg II h\&k resonance line profiles between WASP-12, observed with COS, and three comparison stars, observed with STIS. Two of the comparison stars have effective temperature and age similar to the one of WASP-12. The third comparison star is Procyon, which is known to have a particularly low activity level. Right panel: Position of WASP-12 on the HR diagram assuming three different stellar distances: 265 pc (circle), 295 pc (triangle), and $465 \mathrm{pc}$ (inverted triangle). The dotted, thin full and dashed lines show isochrones from Marigo et al. (2008) corresponding to ages of $1 \mathrm{Gyr}, 2 \mathrm{Gyr}$ and $3 \mathrm{Gyr}$, respectively, encompassing the possible age range of WASP-12. The thick full line is the $2.65 \mathrm{Gyr}$ isochrone we argue being the maximum possible age for WASP-12. Evolutionary tracks from Girardi et al. (2000) for $1.5 \mathrm{M} / M_{\odot}, 1.4 \mathrm{M} / M_{\odot}, 1.3 \mathrm{M} / M_{\odot}$ and $1.2 \mathrm{M} / \mathrm{M}_{\odot}$, from top to bottom, are also shown. Both isochrones and evolutionary tracks assume a metallicity $Z$ of 0.03 . The vertical lines show the WASP-12's temperature range; these lines change from full to dashed below the ZAMS.

plotted as crosses in Fig. 1 (right panel). These suggest an early ingress in the NUVA spectral region.

Lai et al. (2010) and Vidotto et al. (2010) suggested that the early ingress is caused by a bow shock ahead of the planetary orbital motion, formed by the collision of the planet material with the stellar wind.

Li et al. (2010) predicted a loss of material from WASP-12 b, suggesting that this material forms a disk before falling onto the stellar surface, polluting the photosphere. Since we expect the planet metallicity to be higher than that of the star (as in the solar system), this effect would lead to an increased metallicity of the stellar photosphere.

To explore this possibility, we observed WASP-12 using the ESPaDOnS spectropolarimeter at the Canada-France-Hawaii Telescope (CFHT) on the 3rd and 5th of January 2010, in 'polarimetric' mode (see Fossati et al. (2010b) for more details).

Making use of the ESPaDOnS spectra, we performed a detailed parameter determination and abundance analysis, which lead to a set of high precision stellar parameters $\left(T_{\text {eff }}=6250 \pm 100 \mathrm{~K}, \log g=4.2 \pm 0.2, v_{\text {mic }}=1.2 \pm 0.3 \mathrm{~km} \mathrm{~s}^{-1},[\mathrm{Fe} / \mathrm{H}]=0.32 \pm 0.12 \mathrm{dex}\right.$, $\left.Z=0.021 \pm 0.002, v \sin i<4.6 \pm 0.5 \mathrm{~km} \mathrm{~s}^{-1}, v_{\text {macro }}=4.75-7.0 \pm 0.6 \mathrm{~km} \mathrm{~s}^{-1}\right)$ and abundances for 39 ions (see Fossati et al. 2010b). We examined the correlation between the relative abundance of various elements and their condensation temperature, but no clear trend was found. We also compared the WASP-12 abundances with those obtained by other authors on a large number of stars with a similar $T_{\text {eff }}$, concentrating on stars that are not known planet hosts, in the temperature range $6000-6500 \mathrm{~K}$, and with a $[\mathrm{Fe} / \mathrm{H}]>0.0$ dex. The information gathered from this comparison lead to the conclusion that there is the possibility that the WASP-12 abundances of $\mathrm{Na}$, $\mathrm{Si}$, and $\mathrm{Ni}$ do not really match the abundance pattern that the WASP-12 Fe abundance would suggest (see Fossati et al. (2010b) for more details). 


\section{The peculiar stellar activity of WASP-12}

The left panel of Fig. 1 reveals an anomaly in the stellar spectrum: there is a complete lack of the ubiquitous emission cores in the MgII h\&k. This is stunning, given the spectral type and the stellar age. The lack of stellar activity becomes evident in the left panel of Fig. 2, where the Mg II h\&k lines are compared those of other stars with similar temperature and age, taken from the StarCAT archive (Ayres 2010).

It is well known that the chromospheric activity is strongly correlated with the stellar rotational velocity, which for WASP-12 is unknown. Given the very small RossiterMcLaughlin effect (Hebb et al. 2009), it is possible that the low stellar activity of WASP12 might be connected to the low rotational velocity, which could be due to the presence of a global magnetic field. Fossati et al. (2010b) tried to detect the stellar magnetic field from the analysis of the ESPaDOnS Stokes $V$ spectra, but they were able to give only an upper limit of $10 \mathrm{G}$ on the longitudinal component.

It is also possible that the star is passing through a period of low activity; future observations can confirm or reject this hypothesis. The right panel of Fig. 2 excludes stellar ages $>2.65 \mathrm{Gyr}$, given the well established WASP-12 $T_{\text {eff }}$ and metallicity. About half of the main-sequence lifetime remains (see Fossati et al. (2010b) for more details).

Other possibilities to be explored include the lack of the typical signs of stellar activity, such as emission in the cores of the MgII resonance lines which might be absorbed by the material lost by the planet and falling onto the star.

\section{References}

Ayres, T. R. 2010, ApJS, 187, 149

Fossati, L., Haswell, C. A., Froning, C.S., Hebb, L., Holmes, S., Kolb, U., Helling, C., Carter, A., Wheatley, P., Cameron, A.C., Loeillet, B., Pollacco, D., Street, R., Stempels, H. C., Simpson, E., Udry, S., Joshi, Y. C., West, R. G., Skillen, I., \& Wilson, D. 2010, ApJL, 714, 222

Fossati, L., Bagnulo, S., Elmasli, A., Haswell, C. A., Holmes, S., Kochukhov, O., Shkolnik, E. L., Shulyak, D. V., Bohlender, D., Albayrak, B., Froning, C., \& Hebb, L. 2010, ApJ, 720, 872

Girardi, L., Bressan, A., Bertelli, G., \& Chiosi, C. 2000, A\&AS, 141, 371

Hebb, L., Cameron, A. C., Loeillet, B., Pollacco, D., Hébrard, G., Street, R. A., Bouchy, F., Stempels, H.C., Moutou, C., Simpson, E., Udry, S., Joshi, Y.C., West, R. G., Skillen, I., Wilson, D. M., McDonald, I., Gibson, N. P., Aigrain, S., Anderson, D. R., Benn, C. R., Christian, D. J., Enoch, B., Haswell, C. A., Hellier, C., Horne, K., Irwin, J., Lister, T. A., Maxted, P., Mayor, M., Norton, A. J., Parley, N., Pont, F., Queloz, D., Smalley, B., \& Wheatley, P. J. 2009, ApJ, 693, 1920

Lai, D., Helling, C., \& van den Heuvel, E. P. J. 2010, ApJ, 721, 923

Li, S., Miller, N., Lin, D. N. C., \& Fortney, J. J. 2010, Nature, 463, 1054

Marigo, P., Girardi, L., Bressan, A., Groenewegen, M. A. T., Silva, L., \& Granato, G. L. 2008, $A \mathscr{E} A, 482,883$

Osterman, S., et al. 2011, Ap\&SSS, in press

Shkolnik, E., Walker, G. A. H., Bohlender, D. A., Gu, P.-G., \& Kürster, M. 2005, ApJ, 622, 1075

Shulyak, D., Tsymbal, V., Ryabchikova, T., Stütz, Ch., \& Weiss, W. W. 2004, A\&A, 428, 993

Vidotto, A. A., Jardine, M., \& Helling, C. 2010, ApJL, 722, L168 